\title{
ARTICLE OPEN Engineering coherent interactions in molecular nanomagnet dimers
}

Arzhang Ardavan ${ }^{1}$, Alice M Bowen ${ }^{1}$, Antonio Fernandez ${ }^{2}$, Alistair J Fielding ${ }^{2}$, Danielle Kaminski ${ }^{1}$, Fabrizio Moro ${ }^{2}$, Christopher A Muryn ${ }^{2}$, Matthew D Wise ${ }^{3}$, Albert Ruggi ${ }^{3}$, Eric JL Mclnnes ${ }^{2}$, Kay Severin ${ }^{3}$, Grigore A Timco ${ }^{2}$, Christiane R Timmel $^{4}$, Floriana Tuna ${ }^{2}$, George FS Whitehead ${ }^{2}$ and Richard EP Winpenny ${ }^{2}$

Proposals for systems embodying condensed matter spin qubits cover a very wide range of length scales, from atomic defects in semiconductors all the way to micron-sized lithographically defined structures. Intermediate scale molecular components exhibit advantages of both limits: like atomic defects, large numbers of identical components can be fabricated; as for lithographically defined structures, each component can be tailored to optimise properties such as quantum coherence. Here we demonstrate what is perhaps the most potent advantage of molecular spin qubits, the scalability of quantum information processing structures using bottom-up chemical self-assembly. Using $\mathrm{Cr}_{7} \mathrm{Ni}$ spin qubit building blocks, we have constructed several families of two-qubit molecular structures with a range of linking strategies. For each family, long coherence times are preserved, and we demonstrate control over the inter-qubit quantum interactions that can be used to mediate two-qubit quantum gates.

npj Quantum Information (2015) 1, 15012; doi:10.1038/npjqi.2015.12; published online 8 December 2015

\section{INTRODUCTION}

An information processing device whose elements are capable of storing and processing quantum superposition states (a quantum computer) would support algorithms for useful tasks such as searching ${ }^{1}$ and factoring ${ }^{2}$ that are much more efficient than the corresponding classical algorithms, ${ }^{3}$ and would allow efficient simulation of other quantum systems. ${ }^{4}$ One of the key challenges in realizing a quantum computer lies in identifying a physical system that hosts quantum states sufficiently coherently, and provides appropriate interactions for implementing logic operations. ${ }^{5}$ Among the molecular spin systems that have been

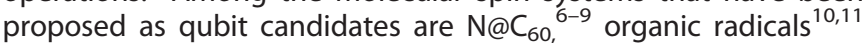
and molecular magnets. ${ }^{12-14}$ We proposed exploiting molecular magnets based on heterometallic antiferromagnetic rings. ${ }^{15,16}$ These systems exhibit a number of favourable features supporting their application as components of a quantum computer: flexibility in their chemical composition allows control over both the total ground-state spin (by modifying the heteroatom) and the carboxylate ligands; ${ }^{17}$ their well-defined internal magnetic excitations may offer mechanisms for efficient single-qubit manipulations; ${ }^{15}$ and the ground-state spin is highly coherent, ${ }^{18}$ particularly when the chemical structure is optimised. ${ }^{19}$

In the context of molecular spin qubits, the simplest conceivable multi-qubit structure is a molecular dimer. This observation has motivated various efforts to synthesise dimers including, for example, $\mathrm{N} @ \mathrm{C}_{60}-\mathrm{N} @ \mathrm{C}_{60}{ }^{20,21}$ radical-radical, ${ }^{10,11}$ $\mathrm{N} @ \mathrm{C}_{60}-$ molecular magnet (Kaminski D. et al. in preparation) and molecular magnet-molecular magnet. ${ }^{22-25}$ However, the design of a dimer specifically to host two-qubit experiments should take account of the importance of three key time scales relative to one another: $T_{2}$, the individual qubit phase relaxation time, must be longest; $h / J$, which is characteristic of the duration of two-qubit gates (where $J$ is the inter-qubit interaction energy and $h$ is Planck's constant) should be intermediate; and the single-qubit manipulation time should be the shortest. In practice, phase relaxation times in heterometallic antiferromagnetic rings are in the $1-10 \mu$ s range at low temperatures, ${ }^{18,19}$ and they can be manipulated in a typical pulsed electron spin resonance (ESR) apparatus on the 10-ns timescale; thus an interaction offering $\mathrm{h} / \mathrm{J}$ in the 100-ns range could be exploited, for example, in a multiqubit experiment to generate controlled entanglement. In this Article we report the synthesis of two families of dimers of the antiferromagnetic ring $\mathrm{Cr}_{7} \mathrm{Ni}$ and pulsed electron spin resonance (ESR) experiments probing the spin coherence times and the intradimer magnetic interactions. We demonstrate that the modular nature of our synthetic approach provides independent control of the molecular nanomagnet components and of the chemical coupling between them. This allows us the flexibility to optimise the physical properties of the dimers with respect to the three key time scales.

\section{METHODS}

Synthesis of multi-qubit molecular structures

The molecular structures of the dimers that we studied are shown in Figure 1. The monomer components are based on $\mathrm{Cr}_{7} \mathrm{Ni}$ rings in which intra-ring nearest-neighbour antiferromagnetic coupling gives rise to a well-defined $S=1 / 2$ ground state. Each dimer family employs a different variety of $\mathrm{Cr}_{7} \mathrm{Ni}$ ring, allowing very different approaches to chemical dimerisation.

The first family (1A, 1C and 1D in Figure 1) is a collection of hybrid [3]rotaxanes. ${ }^{26}$ In each of these compounds two $\mathrm{Cr}_{7} \mathrm{Ni}$ rings are threaded by a rigid organic molecule. There is no covalent bonding between the two

\footnotetext{
${ }^{1}$ Centre for Advanced Electron Spin Resonance, The Clarendon Laboratory, Department of Physics, University of Oxford, Oxford, UK; ${ }^{2}$ School of Chemistry and Photon Science Institute, The University of Manchester, Manchester, UK; ${ }^{3}$ Institut des Sciences et Ingenierie Chimiques, Ecole Polytechnique Fédérale de Lausanne (EPFL), Lausanne, Switzerland and ${ }^{4}$ Centre for Advanced Electron Spin Resonance, Inorganic Chemistry Laboratory, South Parks Road, University of Oxford, Oxford, UK. Correspondence: A Ardavan (arzhang.ardavan@physics.ox.ac.uk)
}

Received 11 May 2015; revised 25 August 2015; accepted 14 September 2015 

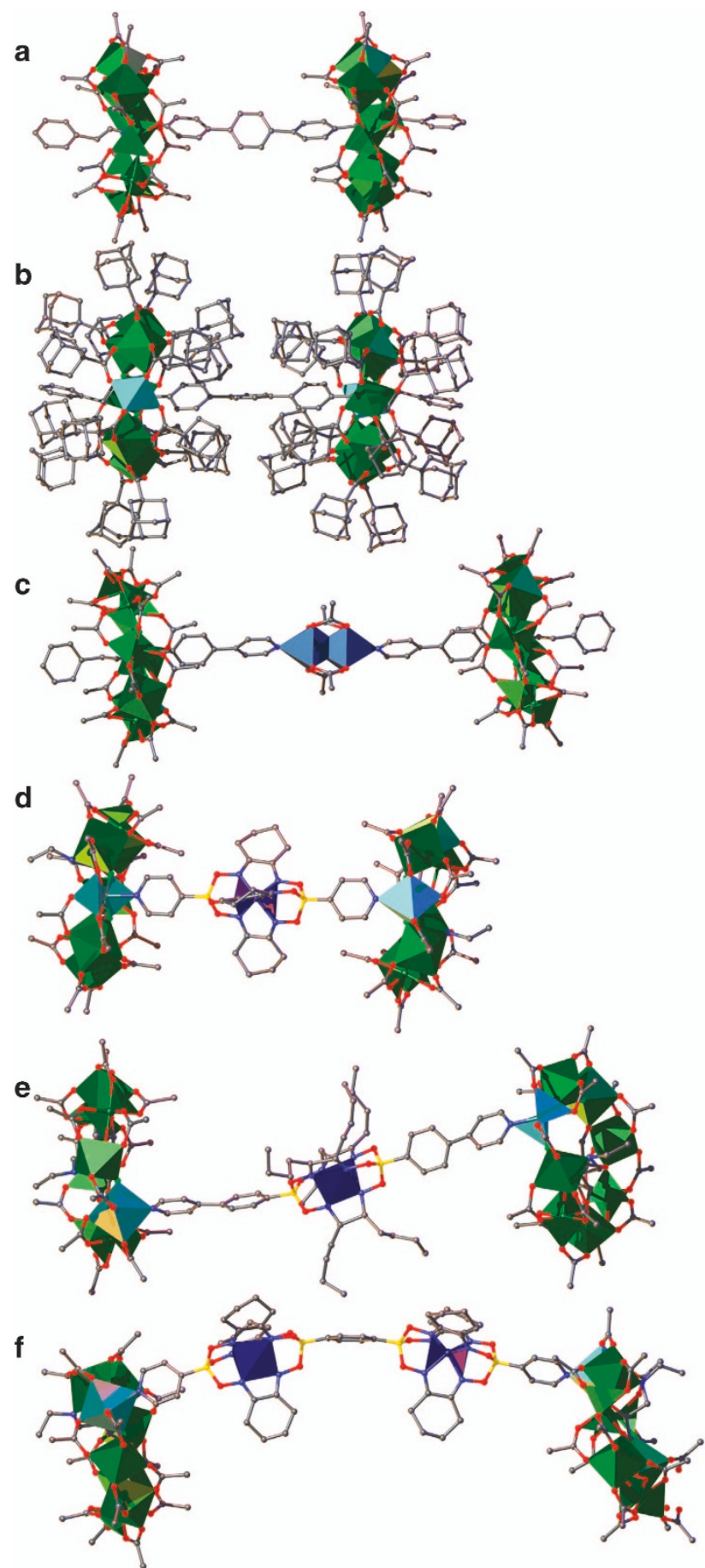

Figure 1. The structure of the two-ring compounds in the crystal. $\mathrm{B}$, yellow balls; $\mathrm{C}$, grey balls; $\mathrm{Cr}$, green octahedra; $\mathrm{Cu}$, blue octahedra; $\mathrm{Fe}$, blue trigonal prisms; $\mathrm{N}$, blue balls; $\mathrm{Ni}$, pale blue octahedra; $\mathrm{O}$, red balls. (a) $\left\{\left[\mathrm{H}_{2} \mathbf{T h} \mathbf{1}\right]\left[\mathrm{Cr}_{7} \mathrm{NiF}_{8}\left(\mathrm{O}_{2} \mathrm{C}^{\mathrm{t}} \mathrm{Bu}\right)_{16}\right]_{2}\right\} \quad \mathbf{1 A}$ (1 B and $\mathbf{1 B d}$ are very similar, differing only by the stopper on the thread); (b) $\left\{\left[\mathrm{H}_{2} \mathrm{Th} 1\right]\left[\mathrm{Cr}_{7} \mathrm{NiF}_{8}\left(\mathrm{O}_{2} \mathrm{CAd}\right)_{16}\right]_{2}\right\} 1 \mathrm{C}$; (c) $\left\{[\mathrm{HTh} 3]\left[\mathrm{Cr}_{7} \mathrm{NiF}_{8}\left(\mathrm{O}_{2} \mathrm{C}^{\mathrm{t}} \mathrm{Bu}\right)_{16}\right]\right\}_{2}$ $\left[\mathrm{Rh}_{2}\left(\mathrm{O}_{2} \mathrm{CMe}\right)_{4}\right]$ 1D; (d) $\left\{\left[\mathrm{Cr}_{7} \mathrm{NiF}_{3}(\mathrm{Etglu})\left(\mathrm{O}_{2} \mathrm{C}^{\mathrm{t} B u}\right)_{15}\right]_{2} \mathrm{~L} 1\right\} \mathbf{2 A}$; (e) $\left\{\left[\mathrm{Cr}_{7} \mathrm{NiF}_{3}\right.\right.$ (Etglu) $\left.\left.\left(\mathrm{O}_{2} \mathrm{C}^{\mathrm{t}} \mathrm{Bu}\right)_{15}\right]_{2} \mathrm{~L} 2\right\}$ 2B; (f) $\left\{\left[\mathrm{Cr}_{7} \mathrm{NiF}_{3}(\text { Etglu })\left(\mathrm{O}_{2} \mathrm{C}^{\mathrm{t}} \mathrm{Bu}\right)_{15}\right]_{2} \mathrm{~L} 3\right\} \mathbf{2 C}$.

rings and the magnetic interaction is expected to be purely dipolar (through space), and modulated by both the length of the threading molecule and the orientation of the dimer with respect to an external magnetic field.

The [3]rotaxane structures, are synthesised by growing the heterometallic rings around rigid thread molecules that contain amine-binding

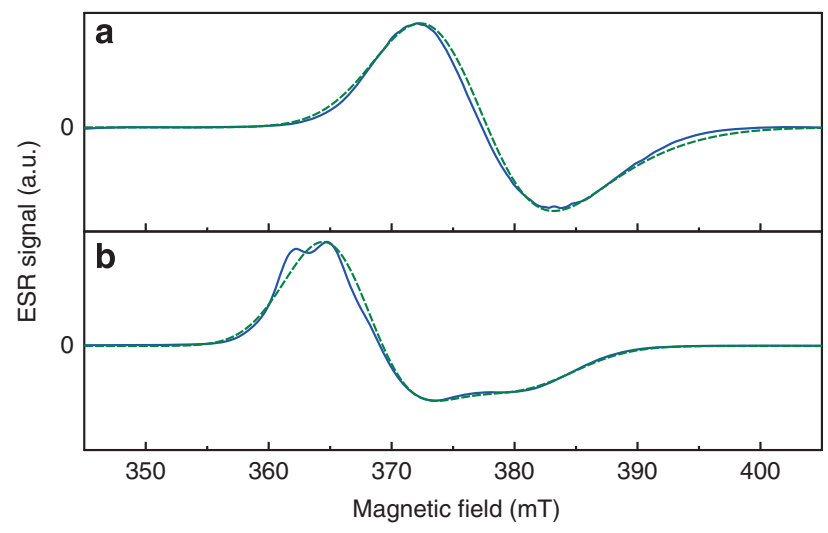

Figure 2. CW ESR spectra of dilute frozen solutions at $2.5 \mathrm{~K}$. (a) X-band CW ESR spectrum of compound $\mathbf{1 A}$ at $9.3878 \mathrm{GHz}$ (blue line) and EasySpin ${ }^{29}$ simulation (green dashed line), yielding parameters $g_{\perp}=1.788$ (strain, 0.02), $g_{\|}=1.748$ (strain, 0.06) A Gaussian FWHM energy broadening (H-strain) of $h \times 250 \mathrm{MHz}$ is included to account for unresolved hyperfine splittings. (b) X-band CW ESR spectrum of $\mathbf{2 A}$ at $9.3892 \mathrm{GHz}$ (blue line) and EasySpin simulation (green dashed line). Simulation parameters: $g_{\perp}=1.83$ (strain, 0.02); $g_{\|}=1.76$ (strain, 0.03 ); $\mathrm{H}$-strain $=h \times 200 \mathrm{MHz}$. The additional splitting, which appears in all family 2 compounds, is probably associated with partially resolved hyperfine interactions.

sites. Initially we studied two closely related threads: $\left[\mathrm{R}-\mathrm{CH}_{2} \mathrm{CH}_{2}-\mathrm{NH}-\mathrm{CH}_{2}-\right.$ $\left.\left(\mathrm{C}_{6} \mathrm{H}_{4}\right)_{3}-\mathrm{CH}_{2}-\mathrm{NH}-\mathrm{CH}_{2} \mathrm{CH}_{2}-\mathrm{R}\right]$ ( $\mathrm{R}=\mathrm{Ph}$, Th1; $\mathrm{R}=\mathrm{CH}_{2} \mathrm{CHMe}_{2}$, Th2). For both threads there are three aromatic rings between the two amines, and they differ only in the stopper on the thread. The reaction to make the [3] rotaxanes involves adding the organic thread to a solution of hydrated chromium trifluoride dissolved in pivalic acid $\left(\mathrm{HO}_{2} \mathrm{C}^{\mathrm{t}} \mathrm{Bu}\right)$; the heterometallic rings then grow around each ammonium cation and the ring is completed by addition of a nickel precursor. We made four related [3]rotaxanes (see Figure 1 and Supplementary Information): $\left\{\left[\mathrm{H}_{2} \mathbf{T h} \mathbf{1}\right]\left[\mathrm{Cr}_{7} \mathrm{NiF}_{8}\left(\mathrm{O}_{2} \mathrm{C}^{\mathrm{t}} \mathrm{Bu}\right)_{16}\right]_{2}\right\}$ 1A; $\left\{\left[\mathrm{H}_{2} \mathrm{Th} 2\right]\left[\mathrm{Cr}_{7} \mathrm{NiF}_{8}\left(\mathrm{O}_{2} \mathrm{C}^{\mathrm{t}} \mathrm{Bu}\right)_{16}\right]_{2}\right\}$ 1B; $\left\{\left[\mathrm{H}_{2} \mathbf{T h 2}\right]\left[\mathrm{Cr}_{7} \mathrm{NiF}_{8}\left(\mathrm{O}_{2} \mathrm{Cd}_{-}{ }^{\mathrm{t}} \mathrm{Bu}\right)_{16}\right]_{2}\right\}$ 1 Bd (with the pivalic acid perdeuterated); $\left\{\left[\mathrm{H}_{2} \mathrm{Th}_{1} 1\right]\left[\mathrm{Cr}_{7} \mathrm{NiF}_{8}\left(\mathrm{O}_{2} \mathrm{CAd}\right)_{16}\right]_{2}\right\} \quad 1 \mathrm{C}$ $\left(\mathrm{HO}_{2} \mathrm{Cad}=1\right.$-adamantanecarboxylic acid) in yields between 17 and $35 \%$.

In the [3]rotaxanes the two $\mathrm{Cr}_{7} \mathrm{Ni}$ rings each contain an octagonal array of seven chromium(III) sites and one nickel(II) site, with each edge of the octagon bridged by a single fluoride and two carboxylates; these molecules are green in colour. The dimer structures can essentially be regarded as an octagonal prism of metal sites. The fluorides lie inside the octagon and form $\mathrm{H}$-bonds to the ammonium of the thread. The distance between the $\mathrm{Cr}_{7} \mathrm{Ni}$ ring centroids is $1.64 \mathrm{~nm}$, whereas the longest metalmetal distance between rings is $1.94 \mathrm{~nm}$. The ring cavity is sufficiently small that a phenyl- or iso-propyl group can act as a stopper for the rotaxane. In the crystal structure the nickel site is disordered about the eight metal sites of the octagon. We made compound $\mathbf{1 B d}$ because we have found that deuterating ligands in $\mathrm{Cr}_{7} \mathrm{Ni}$ monomers can significantly prolong the spin coherence times. ${ }^{18}$ Similarly spin coherence times in isolated rings can be improved by reducing the mobility of nuclear spins in the ligands, ${ }^{19}$ leading us to prepare 1C, which contains carboxylate ligands with rigid adamantyl groups.

Attempts to increase the inter-ring separation by adding aromatic rings to the linker were limited by the low solubility of longer organic chains containing rigid aromatic groups. Instead, we made a new [2]rotaxane by growing the ring around $\mathrm{Ph}-\mathrm{CH}_{2} \mathrm{NH}_{2}-\mathrm{CH}_{2}-\mathrm{C}_{6} \mathrm{H}_{4}-\mathrm{Py}$ Th3 (Py=pyridyl), giving $\left\{[\mathrm{HTh} 3]\left[\mathrm{Cr}_{7} \mathrm{NiF}_{8}\left(\mathrm{O}_{2} \mathrm{C}^{\mathrm{t}} \mathrm{Bu}\right)_{16}\right]\right\}$ (Supplementary Figure $\mathrm{S2}$ ); this [2] rotaxane contains a pyridyl group at the end of the thread and two of these are then coordinated to the axial position of a dimetal tetracarboxylate compound, in this case $\left[\mathrm{Rh}_{2}\left(\mathrm{O}_{2} \mathrm{CMe}\right)_{4}\right]$. This gives a new route to [3] rotaxanes, and results in $\left\{[\mathrm{HTh} 3]\left[\mathrm{Cr}_{7} \mathrm{NiF}_{8}\left(\mathrm{O}_{2} \mathrm{C}^{\mathrm{t}} \mathrm{Bu}\right)_{16}\right]\right\}_{2}\left[\mathrm{Rh}_{2}\left(\mathrm{O}_{2} \mathrm{CMe}\right)_{4}\right]$ 1D (Figure 1c), where there is a distance of $2.5 \mathrm{~nm}$ between the $\mathrm{Cr}_{7} \mathrm{Ni}$ ring centroids of the [3]rotaxane.

The second family of dimers (2A, $\mathbf{2 B}$ and $\mathbf{2 C}$ in Figure 1$)$ involves a chiral $\mathrm{Cr}_{7} \mathrm{Ni}$ ring, $\left[\mathrm{Cr}_{7} \mathrm{NiF}_{3}(\mathrm{Etglu})\left(\mathrm{O}_{2} \mathrm{C}^{\mathrm{t}} \mathrm{Bu}\right)_{15}\left(\mathrm{H}_{2} \mathrm{O}\right)\right]$ (where $\mathrm{H}_{5} \mathrm{Etglu}=\mathrm{N}$-ethyl- $D$ glucamine). ${ }^{27}$ This structure again contains an octagon of metal sites, but at the centre is a penta-deprotonated $N$-ethyl-D-glucamine, which bridges between $\mathrm{Cr}$ sites using the deprotonated alkoxide side-arms. Fluorides bridge three edges of the octagon, and there are again pivalate 
Table 1. Phase relaxation times and two-qubit gate times

\begin{tabular}{lccc}
\hline $\begin{array}{l}\text { Compound (inter-ring } \\
\text { distance) }\end{array}$ & $T_{m}$ (ns) & $x$ & $\begin{array}{c}\text { Two-qubit gate } \\
\text { time (ns) }\end{array}$ \\
\hline 1A $(1.64 \mathrm{~nm})$ & $801 \pm 3$ & $1.615 \pm 0.007$ & 77 \\
1Bd $(1.64 \mathrm{~nm})$ & $3239 \pm 15$ & $1.294 \pm 0.008$ & 84 \\
1C $(1.64 \mathrm{~nm})$ & $2442 \pm 11$ & $1.209 \pm 0.007$ & 82 \\
1D $(2.50 \mathrm{~nm})$ & $543 \pm 8$ & $1.295 \pm 0.014$ & 225 \\
2A $(1.89 \mathrm{~nm})$ & $608 \pm 12$ & $1.261 \pm 0.02$ & 157 \\
2B $(2.71 \mathrm{~nm})$ & $683 \pm 4$ & $1.446 \pm \pm 0.01$ & 400 \\
2C $(3.07 \mathrm{~nm})$ & $543 \pm 18$ & $1.183 \pm 0.03$ & 550 \\
\hline
\end{tabular}

Hahn echo decays measured at $2.5 \mathrm{~K}$ were fitted to a phenomenological stretched exponential function of the form $A$ exp $\left[-\left(T_{\mathrm{m}} / 2 \tau\right)^{x}\right]$ where $2 \tau$ was the total duration of the experiment, and $T_{\mathrm{m}}$ and $x$ were fit parameters ( $x=1$ corresponds to a simple exponential, for which $\left.T_{\mathrm{m}} \equiv T_{2}\right) .{ }^{19} \pi / 2$ - and $\pi$-pulses were, respectively, 140 and $280 \mathrm{~ns}$ long in order to suppress proton ESEEM. Solvents used were protonated toluene (samples 1A, 1B 1C, 2B) or deuterated toluene (1D, 2A, 2C). Concentrations were $100 \mu \mathrm{M}$ (samples 1A, 1B, 1C) or $200 \mu \mathrm{M}$ (samples 1D, 2A, 2B, 2C). The two-qubit gate time corresponds to the first minimum in the oscillatory DEER signal. (Note that for compounds $\mathbf{2 A}, \mathbf{2 B}$, and $\mathbf{2 C}$, the quoted inter-ring distance is a minimum; the rotational degree of freedom for rings in these structures may extend the ring separation.)

groups outside the ring. These molecules are purple in colour. The key difference from the rings in the first family is that there is a substitutionally labile water molecule on the nickel(II) site, which is unique and defined crystallographically. Reacting two such rings with, for example, a linear diimine gives dimers linked via the $\mathrm{Ni}-\mathrm{Ni}$ axis.

Previously we have used simple di-imines, ${ }^{23}$ producing dimers in which we can vary the inter-ring interaction through distance and through the torsion angle within the bridging group. The short di-imine linker leads to inter-ring interaction energies of the order of $h \times 0.3 \mathrm{GHz}$ to $h \times 10 \mathrm{GHz}$, giving rise to large splittings in the continuous wave (CW) ESR spectrum compared with the monomer. ${ }^{23}$ Interactions on this scale would give rise to inter-qubit gate times that are shorter than the duration of single-qubit gates, precluding the possibility of multi-qubit pulsed ESR experiments.

In order to extend the separation of the covalently bound ring dimers, we have employed boronic acid-capped clathrochelate complexes, ${ }^{28}$ which have previously been used as scaffolds to create bipyridyl linkers varying in length from 1.5 to $5.4 \mathrm{~nm}$. The three dimers in this family all contain $\mathrm{Fe}$ (II) (low-spin and diamagnetic in this trigonal prismatic coordination environment) as the divalent metal within a capped tris (dioxime) macrobicycle. We synthesised the clathrochelates as described elsewhere ${ }^{28}$ and mixed them with $\left[\mathrm{Cr}_{7} \mathrm{NiF}_{3}(\mathrm{Etglu})\left(\mathrm{O}_{2} \mathrm{C}^{\mathrm{t}} \mathrm{Bu}\right)_{15}\left(\mathrm{H}_{2} \mathrm{O}\right)\right]$, leading to compounds $\left\{\left[\mathrm{Cr}_{7} \mathrm{NiF}_{3}(\mathrm{Etglu})\left(\mathrm{O}_{2} \mathrm{C}^{\mathrm{t} B u}\right)_{15}\right]_{2} \mathrm{~L} 1\right\} \mathbf{2 A},\left\{\left[\mathrm{Cr}_{7} \mathrm{NiF}_{3}\right.\right.$ (Etglu) $\left.\left.\left(\mathrm{O}_{2} \mathrm{C}^{\mathrm{t}} \mathrm{Bu}\right)_{15}\right]_{2} \mathrm{~L} 2\right\} \quad 2 \mathrm{~B}$ and $\left\{\left[\mathrm{Cr}_{7} \mathrm{NiF}_{3}(\mathrm{Etglu})\left(\mathrm{O}_{2} \mathrm{C}^{\mathrm{t}} \mathrm{Bu}\right)_{15}\right]_{2} \mathrm{~L} 3\right\} \quad \mathbf{2 C}$, shown in Figure 1. Compounds $\mathbf{2 A}$ and $\mathbf{2 C}$ crystallised well, allowing a fullstructure determination. Crystals of 2B were weakly diffracting and only the metal sites could be determined, nevertheless allowing us to estimate the distance between the $\mathrm{Ni}$ sites on the heterometallic rings. The shortest metal-metal contacts in this family are the Ni-Ni distances at 1.89, 2.71 and $3.07 \mathrm{~nm}$ for compounds $\mathbf{2 A}, \mathbf{2 B}$ and $\mathbf{2 C}$ respectively. The longest $\mathrm{Cr}-\mathrm{Cr}$ distances between rings are far greater at $2.24,3.24$ and $3.55 \mathrm{~nm}$ respectively.

\section{Electron spin resonance experiments}

Figure 2 shows $\mathbf{X}$-band $(\sim 9.5 \mathrm{GHz})$ CW ESR spectra of compounds $\mathbf{1 A}$ and $\mathbf{2 A}$ and EasySpin $^{29}$ simulations, from which the spin Hamiltonian parameters may be extracted (see the legend of Figure 2). Within each dimer family, the spectra are very similar; spectra for 1B, 1C, 1D, 2B, and $\mathbf{2} \mathbf{C}$ are shown in Supplementary Figure S1. For both $\mathbf{1 A}$ and $\mathbf{2 A}$, the spectra are characteristic of a spin-1/2 exhibiting an approximately axially anisotropic $g$-factor. The X-band spectrum of $\mathbf{2 A}$ shows more structure on the low-field edge; we believe that this due to partial resolution of hyperfine structure. In both, there is a broadening arising from unresolved hyperfine interactions.

In both compounds the CW spectra are similar to the spectra of the corresponding monomers, ${ }^{18,30}$ indicating that in these compounds the inter-ring magnetic interaction is small compared with the ESR linewidth in energy. This is consistent with the requirement that two-qubit gates should take longer than single-qubit manipulations, a first indication that these dimers are more suitable for quantum information processing experiments than earlier heterometallic ring dimers. ${ }^{23} \mathrm{X}$-band pulsed ESR experiments yield spin coherence times, $T_{m}$ (given in Table 1) that are broadly consistent with the times measured for monomers, ${ }^{19}$ offering reassurance that the formation of complexes and dimers is not intrinsically detrimental to the quantum phase coherence.

The form of the CW spectra imposes an upper bound on the intra-dimer magnetic interaction, but to measure this interaction precisely in this regime requires coherent methods; double electron-electron resonance (DEER, also known as pulsed electron double resonance, PELDOR) ${ }^{31-33}$ is the established method for so doing. Although originally applied to inorganic model systems, ${ }^{32}$ in recent years, DEER has been applied extensively to biological systems. ${ }^{34}$ By modifying pairs of sites in proteins with spin labels such as nitronyl nitroxides, DEER can be used to measure the dipolar interaction, and therefore the distance, between the labels. ${ }^{35}$

DEER techniques exploit the fact that, if there is a magnetic interaction between two spin centres, the coherent precession rate of one of the centres is modified by an inversion of the spin state of the other centre. In practice this is achieved through pulsed ESR sequences using two frequencies, such as the standard four-pulse DEER sequence shown in Figure $3 a .^{33}$ The first two pulses, $\pi / 2-\tau_{1}-\pi$ at frequency $v_{1}$, set up a Hahn echo ${ }^{31}$ at a time $2 \tau_{1}$ on one of the spins. As this spin continues to precess, a 'pump' $\pi$ pulse at a second frequency $v_{2}$ at time $T$ (relative to the $v_{1}$ echo) inverts the second spin, thus modifying the effective magnetic field (and therefore the precession rate) experienced by the first spin. A subsequent refocusing $\pi$ pulse at frequency $v_{1}$ a time $\tau_{2}$ after the first echo generates a second echo at a time $2 \tau_{1}+2 \tau_{2}$, with an amplitude that depends on the time at which the $v_{2}$ inversion pulse was applied and on the strength of the interaction between the two spins. Observing this echo as a function of the time, $T$, of the pump pulse reveals oscillations at the frequency corresponding to the magnetic interaction energy between the two centres. A three-pulse variant, shown in Figure $3 b,{ }^{32}$ has the advantage of being shorter and having fewer pulses, thus enhancing the amplitude of the measured echo, particularly for systems in which the spin coherence times are short. The disadvantage is that for short times $T$, the $v_{2}$ pump pulse overlaps the coherence-generating $v_{1} \pi / 2$ pulse, distorting the spectra around the time $T=0$ [33].

This description of DEER depends on some heterogeneity between the two spin centres in the molecule under study, so that the frequencies $v_{1}$ and $v_{2}$ are resonant with different centres. We performed DEER experiments at low temperatures $(2.5 \mathrm{~K}$, to maximise spin coherence times) on dilute frozen solutions of dimers $(0.1-0.2 \mathrm{mM}$, to minimise interdimer dipolar interactions). In this case differences between the two heterometallic rings may arise from conformational flexibility. In the first family, the [3] rotaxane dimers, there may be variation in the position of the $\mathrm{Ni}$ centre between the two rings, or some slight bending of the stiff threading molecule. In the second family, the covalently bound dimers, the rings could rotate about the axis defined by the linker. In either case, the distortions of individual rings (giving rise to $g$-strains) or variations in configurations of hyperfine-coupled nuclei (resulting in variations of the effective local magnetic field) offer asymmetry between the two rings within a dimer.

The CW spectra in Figure 1 extend over a magnetic field range of about $30 \mathrm{mT}$ at X-band (about $9.5 \mathrm{GHz}$ ), corresponding to an absorption spectrum at a fixed magnetic field covering about $750 \mathrm{MHz}$, as plotted in Figure 3c. Intervals within this energy range can be identified with orientationally selected sub-populations of rings. Thus, microwave pulses at different frequencies within the spectrum excite orientations selectively, shown as coloured regions on spheres in Figure 3d. We anticipate that, notwithstanding a degree of flexibility, the orientations of two rings within a single dimer should be reasonably well correlated. With this in mind, we choose to separate $v_{1}$ and $v_{2}$ by a frequency that is small compared to the total width of the spectrum, so that they excite neighbouring orientational sub-populations of rings.

In practice, the response of the microwave resonator used in the experiment is not uniform over the frequency range of the absorption line. Instead we used fixed frequencies for $v_{1}$ and $v_{2}$, and we achieved orientation selection by adjusting the external magnetic field to bring the appropriate part of the absorption spectrum into resonance with the applied pulses. We used detection pulses (at frequency $v_{1}$ ) of $40 \mathrm{~ns}$ and pump pulses $\left(v_{2}\right)$ of $24 \mathrm{~ns}$, separated in frequency by $80 \mathrm{MHz}$. 

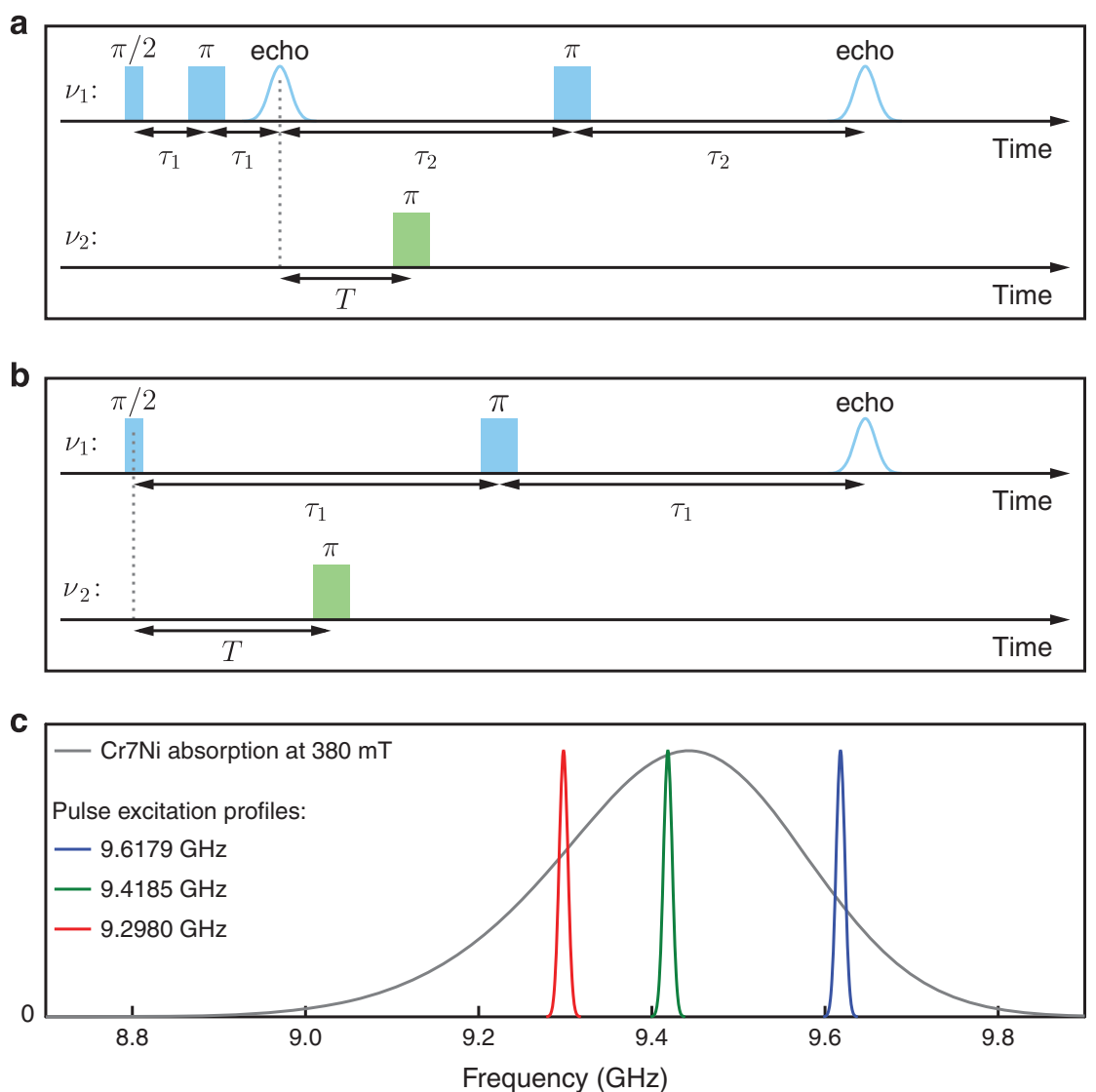

d

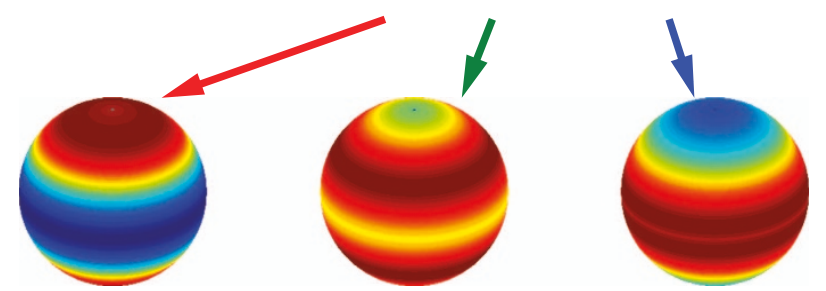

Figure 3. Principle of the DEER experiment. (a) 4-pulse DEER sequence. (b) 3-pulse DEER sequence. (c) ESR absorption spectrum as a function of energy (grey), and excitation profiles for pulses applied resonant with different parts of the absorption spectrum (red, green and blue), (d) Ring orientations relative to the vertical external magnetic field excited by the pulses in c owing to $g$-factor anisotropy and strain (red: high excitation, blue: low excitation).

\section{RESULTS}

Figure 4 shows typical DEER spectra taken on compound $\mathbf{1 A}$. The left panel shows the amplitude of the final echo as a function of the time, $T$, of the pump pulse for three different orientation selections. Open circles are raw data; the solid curves are filtered using standard tools (the Matlab package DeerAnalysis ${ }^{36}$ ) to smooth the data and to remove oscillations (electron spin echo envelope modulation, or ESEEM ${ }^{18,31}$ ) associated with couplings to proton nuclear moments. To the right of each trace, a spherical intensity map indicates the range of orientations probed at the given applied magnetic field and experimental detection frequency. The right panel shows the Fourier transforms of the time-domain data from the left panels (both raw data, open circles, and filtered data, solid lines).

The dependence of the DEER signal on magnetic field (or, equivalently, orientational sub-population) is strong, owing to several contributory factors. First, the inter-ring dipolar interaction depends on the orientation, $\theta$, of the dimer inter-ring axis with respect to the magnetic field as $\left(3 \cos ^{2} \theta-1\right)$. It therefore varies significantly as a function of the dimer orientation and passes through zero at the magic angle, $\theta \approx 54^{\circ}$. Second, the magnitude by which the detected echo is modulated in the DEER experiment depends on the proportion of dimers excited initially by $v_{1}$ that is also excited, at the other end, by $v_{2}$.

In a magnetic field of $377.84 \mathrm{mT}$ (lowest trace, blue), dimers whose inter-spin axes are perpendicular to the field are predominantly excited. Within this sub-population the range of inter-ring magnetic dipolar interactions is strongly peaked around a single value, giving rise to clearly defined oscillations with a period of about $155 \mathrm{~ns}$. The Fourier transform, which represents the distribution of coupling strengths within the selected orientational sub-population, is correspondingly sharply peaked at about $6.5 \mathrm{MHz}$. The first minimum in the time-domain data, occurring at about $77 \mathrm{~ns}$, is the duration for inversion of the state of one of the qubits under the influence of the second. This evolution of the state of one qubit conditional on the state of the other is exactly the kind of physical interaction that allows for implementation of multi-qubit quantum logic; in our case, the time to the first minimum corresponds to the duration of a twoqubit conditional phase gate. We note that this time satisfies the criteria that we identified above for two-qubit devices: the twoqubit gate time (of order $100 \mathrm{~ns}$ ) lies between the single-qubit 

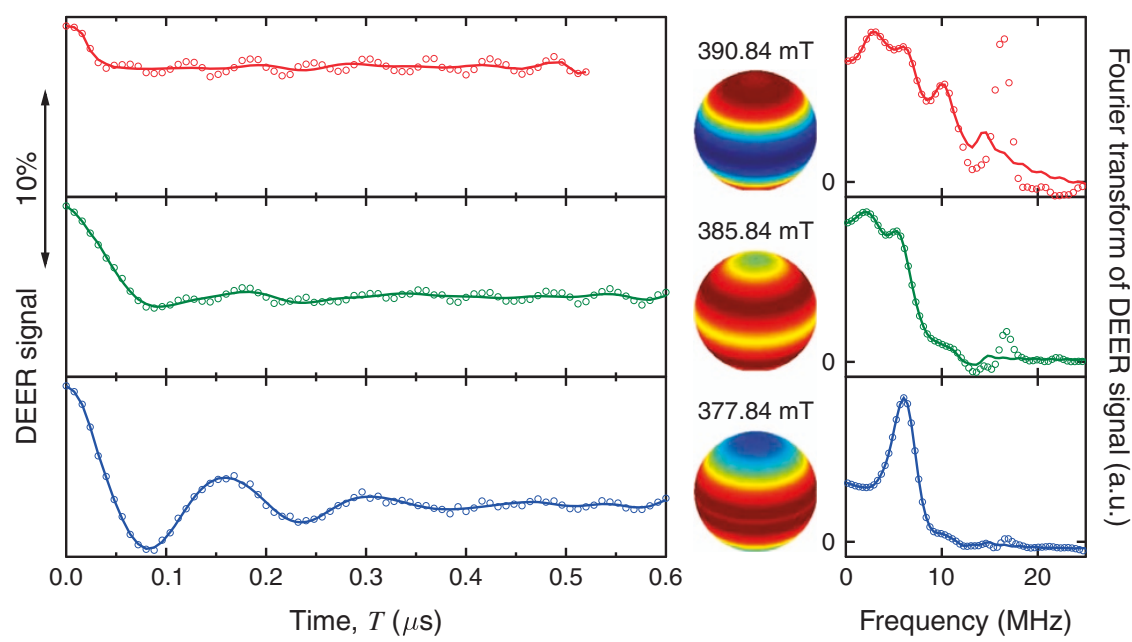

Figure 4. DEER data for sample 1A. The detection and excitation frequencies were, respectively, $v_{1}=9.5632 \mathrm{GHz}, v_{2}=v_{1}-0.08 \mathrm{GHz}=9.4832 \mathrm{GHz}$. The temperature was $2.5 \mathrm{~K}$. The DEER signal was recorded as a function of the time of the $v_{1}$ pump pulse (left panels) at three magnetic fields, each corresponding to an orientational sub-population indicated by the intensity maps on spheres. The Fourier transforms of the timedomain data are shown on the right. Open circles show raw data; solid lines indicate filtered data. The principal difference between the unfiltered data and the filtered data is the absence in the latter of a component at about $18 \mathrm{MHz}$ arising from proton ESEEM.
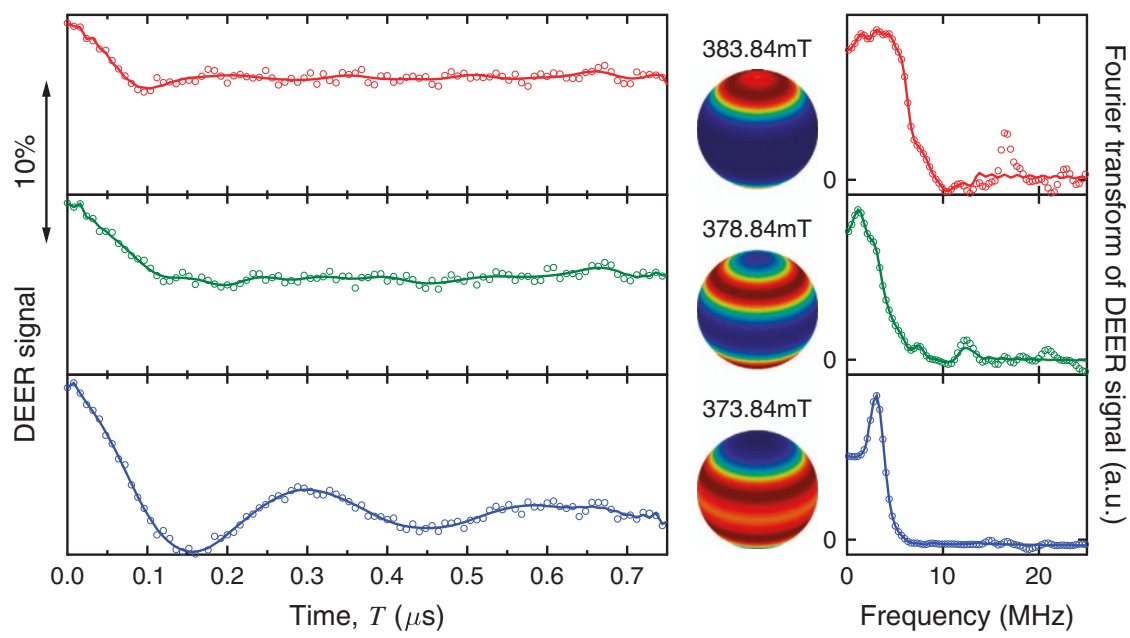

Figure 5. DEER data for sample 2A. The detection and excitation frequencies were, respectively, $v_{1}=9.5260 \mathrm{GHz}$, $v_{2}=v_{1}-0.08 \mathrm{GHz}=9.4460 \mathrm{GHz}$.

gate time (of order $10 \mathrm{~ns}$ ) and the phase relaxation time (in the microsecond range).

The sub-population excited at a magnetic field of $385.84 \mathrm{mT}$ (middle trace, green) is comprised of dimers whose inter-ring axes lie at orientations peaked in between parallel and perpendicular to the external magnetic field. The distribution of orientations includes a significant proportion close to the magic angle at which the dipolar interaction strength goes through zero, resulting in a broad distribution of interaction strengths and an almost monotonic dependence of the time-domain DEER signal on $T$.

At $390.84 \mathrm{mT}, v_{1}$ excites principally rings whose planes are perpendicular to the magnetic field (i.e. those whose effective $g$-factor is close to $\left.g_{\|}\right)$. Deviations from this orientation lead to a greater contribution to the effective $g$-factor from the larger $g_{\perp}$, and therefore to a higher resonant frequency. Thus, in dimers that are orientationally selected by the excitation of one ring at frequency $v_{1}, v_{2}$ (which is lower in frequency than $v_{1}$ ) is comparatively unlikely to excite the other ring. This gives rise directly to the comparatively weak modulation in the time-domain trace at $390.84 \mathrm{mT}$. The inter-spin axes of this sub-population of dimers are aligned along the magnetic field direction, maximising the orientation-dependence of the dipolar interaction (i.e., $\theta \approx 0^{\circ}$ ). Correspondingly, the time-domain signal evolves comparatively rapidly at short times and the Fourier transform contains components at high frequencies.

Figure 5 shows the equivalent DEER data for a sample from the second family, 2A. As for sample 1A, the DEER signal reveals well-defined oscillations and a strongly peaked distribution of inter-ring couplings for populations of dimers whose axes are close to perpendicular to the magnetic field, and broader coupling distributions and weaker modulations for populations at other orientations. However, compared with 1A, 2A exhibits coupling strengths that are smaller by a factor of about two (the first minimum in the time-domain data at $380.84 \mathrm{mT}$ occurs at about $157 \mathrm{~ns}$; the peak in the Fourier transform is at $3.3 \mathrm{MHz}$ ). This is broadly accounted for by the larger separation of the rings in $\mathbf{2 A}$ (which is somewhat extended over the $\mathrm{Ni}-\mathrm{Ni}$ distance of $1.89 \mathrm{~nm}$ by the rotational degree of freedom of the rings about the linker 
axis) than in $\mathbf{1 A}$ (the centroids of whose rings are separated by $1.6 \mathrm{~nm})$, and the fact that the dipolar interaction varies with separation, $r$, as $1 / r^{3}$.

These trends are reflected in the DEER data for all of the compounds shown in Figure 1; the time-domain data and Fourier transforms for compounds other than $\mathbf{1 A}$ and $\mathbf{2 A}$ are presented in the Supplementary Figures S2 to S6, and the two-qubit gate times are listed in Table 1. There is a direct correlation between the interaction strength and the inter-ring separation, with larger separations giving rise to weaker interactions. The two-qubit gate times reach about $550 \mathrm{~ns}$ for the longest dimers. The interactions are weaker in the the second family than in the first, owing to the extra degree of freedom in the structure: rings that are attached to the linker through a single point on the rim of the ring are free to rotate about the linker axis, allowing a greater inter-ring separation.

We note also that our strategy allows for enhancement of the spin coherence times independently of the inter-ring interaction strength. In earlier studies of monomers, ${ }^{19}$ we found that deuteration and immobilisation of nuclear spins in the structure significantly improve the coherence. Thus, in compounds $\mathbf{1 A} \mathbf{A} \mathbf{B d}$ and $1 \mathrm{C}$, while the similar linkers ensure that $h / J$ is similar for each, deuteration (1Bd) and confinement of hydrogen nuclei to rigid adamantyl groups $(\mathbf{1 C})$ result in extensions of the coherence times by factors of about four and three, respectively.

\section{DISCUSSION}

From the data presented here, we may draw several conclusions supporting the assertion that molecular spin systems may form useful components in future quantum technologies. Molecular magnets offer building blocks which, through supramolecular chemistry, can be assembled into multi-qubit structures. Identifying strict criteria for the relative timescales for single-qubit manipulations, two-qubit interactions and phase memory times, we have designed several families of structures to optimise the prospects of hosting two-qubit devices. For example, in compound $1 \mathrm{~A}$, the phase coherence time of about $800 \mathrm{~ns}$ significantly exceeds the two-qubit gate time of $77 \mathrm{~ns}$, and both are much longer than the typical single-qubit manipulation time of about 10 ns. Thus, we build upon the wide range of proposals to exploit molecular nanomagnets in quantum information by demonstrating for the first time coherent manipulation of dimers of such species, including multi-frequency experiments probing the inter-qubit interaction, and by showing that we can chemically tune the interaction strength to meet architectural requirements. This prepares the path for experiments demonstrating controlled entanglement operations (as opposed to the observation of entanglement as an inherent property of a singlet ground state of two coupled spins ${ }^{37}$ ), and other two-qubit manipulations.

\section{ACKNOWLEDGEMENTS}

This work was supported by the EPSRC through the National EPR Service and grants $\mathrm{EP} / \mathrm{H} 012613 / 1, \mathrm{EP} / \mathrm{H} 011714 / 1$, and EP/L018470/1 and EP/K039547/1.

\section{CONTRIBUTIONS}

AA, EJLM, CRT and REPW formulated the project. AF and GAT prepared the compounds, using clathrochelates prepared by MDW, AR and KS. GFSW and CAM performed the X-ray structure analysis. AMB, AJF, DK, FM and FT measured ESR data. $A M B$ and $D K$ analysed and interpreted data presented here. AA wrote the manuscript with substantial input from EJLM and REPW.

\section{COMPETING INTERESTS}

The authors declare no conflict of interest.

\section{REFERENCES}

1. Grover LK. Quantum mechanics helps in searching for a needle in a haystack. Phys Rev Lett 1997; 79: 325-328.

2. Shor PW. Polynomial-time algorithms for prime factorization and discrete logarithms on a quantum computer. SIAM J Comput 1997; 26: 1484-1509.

3. Nielsen MA \& Chuang IL. Quantum Computation and Quantum Information. Cambridge University Press: Cambridge, 2000.

4. Feynman RP. Simulating physics with computers. Int J Theor Phys 1982; 21: 467-488.

5. DiVincenzo DP. The physical implementation of quantum computation. Fortsc Phys 2000; 48: 771-783.

6. Almeida Murphy T, Pawlik Th, Weidinger A, Höhne M, Alcala R \& Paeth J-M. Observation of atomlike nitrogen in nitrogen-implanted $C_{60}$. Phys Rev Lett 1996; 77: $1075-1078$.

7. Harneit W. Fullerene-based electron spin quantum computer. Phys Rev A 2002; 65: 032322.

8. Benjamin SC, Ardavan A, Briggs GAD, Britz DA, Gunlyke D, Jefferson J et al. Towards a fullerene-based quantum computer. J Phys Condens Matter 2006; 18: S867.

9. Morton JJL, Tyryshkin AM, Ardavan A, Benjamin SC, Porfyrakis K, Lyon SA et al. Bang-bang control of fullerene qubits using ultrafast phase gates. Nat Phys 2006; 2: $40-43$.

10. Sato K, Nakazawa S, Rahimi R, Ise T, Nishida S, Yoshino T et al. Molecular electronspin quantum computers and quantum information processing: pulse-based electron magnetic resonance spin technology applied to matter spin-qubits. J Mater Chem 2009; 19: 3739-3754.

11. Ayabe K, Sato K, Nishida S, Ise T, Nakazawa S, Sugisaki K et al. Pulsed electron spin nutation spectroscopy of weakly exchange-coupled biradicals: a general theoretical approach and determination of the spin dipolar interaction. Phys Chem Chem Phys 2012; 14: 9137-9148.

12. Leuenberger MN \& Loss D. Quantum computing in molecular magnets. Nature 2001; 410: 789-793.

13. Tejada J, Chudnovsky EM, del Barco E, Hernandez JM \& Spiller TP. Magnetic qubits as hardware for quantum computers. Nanotechnology 2001; 12: 181.

14. Meier F, Levy J \& Loss D. Quantum computing with spin cluster qubits. Phys Rev Lett 2003; 90: 047901.

15. Troiani F, Ghirri A, Affronte M, Carretta S, Santini P, Amoretti G et al. Molecular engineering of antiferromagnetic rings for quantum computation. Phys Rev Lett 2005; 94: 207208.

16. Ardavan A \& Blundell SJ. Storing quantum information in chemically engineered nanoscale magnets. J Mater Chem 2009; 19: 1754-1760.

17. Larsen FK, McInnes EJL, El Mkami H, Overgaard J, Piligos S, Rajaraman G et al. Synthesis and characterization of heterometallic $\left\{\mathrm{Cr}_{7} \mathrm{M}\right\}$ wheels. Angew Chem Int Ed 2003; 42: 101-105.

18. Ardavan A, Rival O, Morton JJL, Blundell SJ, Tyryshkin AM, Timco GA et al. Will spin relaxation times in molecular magnets permit quantum information processing? Phys Rev Lett 2007; 98: 057201

19. Wedge CJ, Timco GA, Spielberg ET, George RE, Tuna F, Rigby S et al. Chemical engineering of molecular qubits. Phys Rev Lett 2012; 108: 107204.

20. Farrington BJ, Jevric M, Rance GA, Ardavan A, Khlobystov AN, Briggs GAD et al. Chemistry at the nanoscale: synthesis of an $\mathrm{N} @ \mathrm{C}_{60}-\mathrm{N} @ \mathrm{C}_{60}$ endohedral fullerene dimer. Angew Chem Int Ed 2012; 51: 3587-3590.

21. Plant SR, Jevric M, Morton JJL, Ardavan A, Khlobystov AN, Briggs GAD et al. A two-step approach to the synthesis of $\mathrm{N} @ \mathrm{C}_{60}$ fullerene dimers for molecular qubits. Chem Sci 2013; 4: 2971-2975.

22. Hill S, Edwards RS, Aliaga-Alcalde N \& Christou G. Quantum coherence in an exchange-coupled dimer of single-molecule magnets. Science 2003; 302: 1015-1018.

23. Faust TB, Bellini V, Candini A, Carretta S, Lorusso G, Allan DR et al. Chemical control of spin propagation between heterometallic rings. Chem Eur J 2011; 17: 14020-14030.

24. Luis F, Repollés A, Martínez-Pérez MJ, Aguilà D, Roubeau O, Zueco D et al. Molecular prototypes for spin-based CNOT and SWAP quantum gates. Phys Rev Lett 2011; 107: 117203

25. Aguila D, Barrios LA, Velasco V, Roubeau O, Repollés A, Alonso PJ et al. Heterodimetallic [ $\mathrm{LnLn}^{\prime}$ ] lanthanide complexes: toward a chemical design of two-qubit molecular spin quantum gates. J Am Chem Soc 2014; 136 $14215-14222$.

26. Lee C-F, Leigh DA, Pritchard RG, Schultz D, Teat SJ, Timco GA et al. Hybrid organic-inorganic rotaxanes and molecular shuttles. Nature 2009; 458 : 314-318.

27. Garlatti E, Albring MA, Baker ML, Docherty RJ, Mutka H, Guidi T et al. A detailed study of the magnetism of chiral $\left\{\mathrm{Cr}_{7} \mathrm{M}\right\}$ rings: an investigation into parameterization and transferability of parameters. J Am Chem Soc 2014; 136 9763-9772. 
28. Wise MD, Ruggi A, Pascu M, Scopelliti R \& Severin K. Clathrochelate-based bipyridyl ligands of nanoscale dimensions: easy-to-access building blocks for supramolecular chemistry. Chem Sci 2013; 4: 1658-1662.

29. Stoll S \& Schweiger A. EasySpin, a comprehensive software package for spectral simulation and analysis in EPR. J Magn Reson 2006; 178: 42-55.

30. Timco GA, McInnes EJL, Pritchard RG, Tuna F \& Winpenny REP. Heterometallic rings made from chromium stick together easily. Angew Chem Int Ed 2008; 47: 9681-9684.

31. Milov AD, Ponomarev AB \& Tsvetkov Yu. Electron-electron double resonance in electron spin echo: model biradical systems and the sensitized photolysis of decalin. Chem Phys Lett 1984; 110: 67-72.

32. Pannier M, Veit S, Godt A, Jeschke G \& Spiess HW. Dead-time free measurement of dipole-dipole interactions between electron spins. J Magn Reson 2000; 142: 331-340.

33. Schweiger A \& Jeschke G. Principles of Pulse Electron Paramagnetic Resonance. Oxford University Press: Oxford, UK, 2001.

34. Schiemann O \& Prisner TF. Long-range distance determinations in biomacromolecules by EPR spectroscopy. Quart Rev Biophys 2007; 40: 1-53.
35. Jeschke G \& Polyhach Y. Distance measurements on spin-labelled biomacromolecules by pulsed electron paramagnetic resonance. Phys Chem Chem Phys 2007; 9: 1895-1910.

36. Jeschke $G$, Chechik V, lonita $P$, Godt A, Zimmermann $H$, Banham J et al. DeerAnalysis2006-a comprehensive software package for analyzing pulsed ELDOR data. Appl Magn Reson 2006; 30: 473-498.

37. Candini A, Lorusso G, Troiani F, Ghirri A, Carretta S, Santini P et al. Entanglement in supramolecular spin systems of two weakly coupled antiferromagnetic rings (purple-Cr ${ }_{7} \mathrm{Ni}$ ). Phys Rev Lett 2010; 104: 037203.

\section{cc) (i)}

This work is licensed under a Creative Commons Attribution 4.0 International License. The images or other third party material in this article are included in the article's Creative Commons license, unless indicated otherwise in the credit line; if the material is not included under the Creative Commons license, users will need to obtain permission from the license holder to reproduce the material. To view a copy of this license, visit http://creativecommons.org/licenses/ by/4.0/

Supplementary Information accompanies the paper on the npj Quantum Information website (http://www.nature.com/npjqi) 